\title{
Effectiveness and safety of Bifidobacterium and berberine in human hyperglycemia and their regulatory effect on the gut microbiota: a multi-center, double-blind, randomized, parallel-controlled study
}

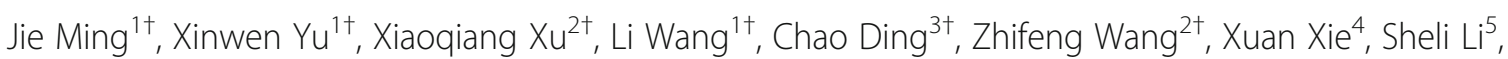
Wenjuan Yang ${ }^{6,7}$, Shu Luo ${ }^{8}$, Qingzhen $\mathrm{He}^{9}$, Yafang Du ${ }^{10}$, Zhufang Tian ${ }^{11}$, Xiling Gao ${ }^{12}$, Kaiyan Ma ${ }^{13}$, Yujie Fang ${ }^{1}$, Chen $\mathrm{Li}^{14}$, Jiajun Zhao ${ }^{15^{*}}$, Xiaokai Wang ${ }^{2^{*}}$ and Qiuhe $\mathrm{Ji}^{i^{*}}$ (D)

\begin{abstract}
Background: Berberine and Bifidobacterium have been reported to improve glucose tolerance in people with hyperglycemia or other metabolic disorders. This study aimed to assess the hypoglycemic effect and the regulation of the gut microbiota caused by berberine and Bifidobacterium and the possible additive benefits of their combination.

Methods: This was an 18-week, multi-center, randomized, double-blind, parallel-controlled study of patients newly diagnosed with hyperglycemia. After a 2-week run-in period, 300 participants were randomly assigned to the following four groups for 16 weeks of treatment: berberine (Be), Bifidobacterium (Bi), berberine and Bifidobacterium (BB), and placebo group. The primary efficacy endpoint was the absolute value of fasting plasma glucose (FPG) compared with baseline after 16 weeks of treatment.
\end{abstract}

\footnotetext{
* Correspondence: qiuheji@hotmail.com; jqiuhe@fmmu.edu.cn;

wangxiaokai@aimigene.com; jjzhao@sdu.edu.cn

†Jie Ming, Xinwen Yu, Xiaoqiang Xu, Li Wang, Chao Ding and Zhifeng Wang contributed equally to this work.

${ }^{15}$ Department of Endocrinology and Metabolism, Shandong Provincial Hospital Affiliated to Shandong University, Jinan 250021, China

${ }^{2}$ Aimigene Institute, Shenzhen 518063, China

${ }^{1}$ Endocrinology Research Center, Department of Endocrinology and

Metabolism, Xijing Hospital, Fourth Military Medical University, Xi'an 710032,

China

Full list of author information is available at the end of the article
}

(c) The Author(s). 2021 Open Access This article is licensed under a Creative Commons Attribution 4.0 International License, which permits use, sharing, adaptation, distribution and reproduction in any medium or format, as long as you give appropriate credit to the original author(s) and the source, provide a link to the Creative Commons licence, and indicate if changes were made. The images or other third party material in this article are included in the article's Creative Commons licence, unless indicated otherwise in a credit line to the material. If material is not included in the article's Creative Commons licence and your intended use is not permitted by statutory regulation or exceeds the permitted use, you will need to obtain permission directly from the copyright holder. To view a copy of this licence, visit http://creativecommons.org/licenses/by/4.0/ The Creative Commons Public Domain Dedication waiver (http://creativecommons.org/publicdomain/zero/1.0/) applies to the data made available in this article, unless otherwise stated in a credit line to the data. 
Results: Between October 2015 and April 2018, a total of 297 participants were included in the primary analysis. Significant reductions of FPG were observed in the Be and BB groups compared with the placebo group, with a least square (LS) mean difference of $-0.50,95 \% \mathrm{Cl}[-0.85,-0.15] \mathrm{mmol} / \mathrm{L}$, and $-0.55,95 \% \mathrm{Cl}[-0.91,-0.20]$ $\mathrm{mmol} / \mathrm{L}$, respectively. The Be and BB groups also showed significant reductions in 2-h postprandial plasma glucose. A pronounced decrease in HbA1c occurred in the BB group compared to the placebo group. Moreover, compared with the Bi and placebo groups, the Be and BB groups had more changes in the gut microbiota from the baseline.

Conclusions: Berberine could regulate the structure and function of the human gut microbiota, and Bifidobacterium has the potential to enhance the hypoglycemic effect of berberine. These findings provide new insights into the hypoglycemic potential of berberine and Bifidobacterium.

Trial registration: ClinicalTrials.gov, NCT03330184. Retrospectively registered on 18 October 2017

Keywords: Bifidobacterium, Berberine, Gut microbiota, Hyperglycemia

\section{Background}

Type 2 diabetes (T2D) is one of the metabolic diseases with increasing prevalence worldwide [1]. Pharmacological intervention for T2D remains the gold treatment standard if lifestyle modification fails. Although various anti-diabetic drugs have been developed and used to treat diabetes, side effects and long-term efficacy pose frequent challenges [2]. Thus, safer and more effective medications are urgently needed. A series of studies suggest a link between the gut microbiota and human metabolic health. Mounting evidence has indicated that the gut microbiota affects the pharmacology of anti-diabetic drugs, and that in return, the metabolic products induced by these drugs transform the structure and function of the gut microbiota $[3,4]$. Current studies have shown that the gut microbiota has become the target for anti-diabetic drugs.

Berberine is a major constituent of traditional Chinese medicine, Coptis chinensis, which is usually used as an antibiotic to treat diarrhea [5]. Existing studies have revealed its therapeutic effects on hyperglycemia and dyslipidemia in humans [6]. However, the mechanism of berberine is still unknown. Berberine administered orally has poor intestinal absorption and bioavailability [7] and low serum concentration [8], resulting in concerns over its effect on the gut microbiota. Previous animal studies have demonstrated that berberine may regulate the structure and function of the gut microbiota and alleviate insulin resistance by increasing the abundance of beneficial microbiota [9] such as Bifidobacterium [10], producing short-chain fatty acids [11], reducing the biosynthesis of branched-chain amino acids [12], altering the microbial bile acid metabolism [13], inhibiting the expression of intestinal inflammatory cytokines [14, 15], and improving the gut microbiota energy metabolism [16]. However, the evidence for the regulation of the human gut microbiota by berberine is limited. Some human studies reported the gastrointestinal reactions that occur after berberine treatment, including diarrhea and constipation, which may also be related to the gut microbiota [17-19]. In addition, the gut microbiota could enhance the biological activity of berberine by converting it into a more absorbable form, dihydroberberine [20]. Therefore, we hypothesized that the concomitant use of probiotics with berberine may improve the treatment effect.

Bifidobacterium is a widely used probiotic supplement. Population studies have indicated that oral supplementation of probiotics including Bifidobacterium could improve metabolic disorders such as T2D [21], gestational diabetes [22], excessive weight gain or obesity [23], metabolic syndrome, and non-alcoholic fatty liver [24, 25]. Berberine and the common anti-diabetic drugs acarbose and metformin have been shown to increase the abundance of Bifidobacterium after treatment $[3,4,10]$. However, due to the lack of studies about Bifidobacterium used alone, its effect on humans remains unclear. It is also not known whether Bifidobacterium can be fostered by other anti-diabetic medicines used for glucose control or whether the underlying mechanism involves regulating the intestinal environment to achieve a better hypoglycemic effect.

Therefore, this study was conducted to observe the hypoglycemic effect of berberine and Bifidobacterium, to verify the potential intestinal mechanism of berberine and Bifidobacterium, and to discern the possible benefits of adding Bifidobacterium to a berberine regimen.

\section{Methods \\ Study design}

The design of this study has been previously published, as a full study protocol [26]. Briefly, we performed a multi-center, randomized, double-blind, parallelcontrolled study on newly diagnosed patients with hyperglycemia, which included a run-in period of 2 weeks and a treatment period of 16 weeks. This multicenter study was conducted in 10 tier 2 or 3 hospitals in Shaanxi province, China, between October 2015 and 
April 2018, and was approved by the independent Ethics Committee or institutional review board at each hospital. All of the participants provided written informed consent before study entry. This study was registered with ClinicalTrials.gov, NCT03330184.

\section{Participants}

This study enrolled individuals aged 18-70 years with hyperglycemia, diagnosed by oral glucose tolerance test (the V1 stage was defined by fasting plasma glucose (FPG) of $5.6 \leq \mathrm{FPG}<8.0 \mathrm{mmol} / \mathrm{L}$, while the V2 stage was defined by $6.1 \leq \mathrm{FPG}<8.0 \mathrm{mmol} / \mathrm{L}$ or a 2 -hour postprandial plasma glucose [2-hr PPG] of $7.8 \leq 2$-hr PPG $<17 \mathrm{mmol} / \mathrm{L}$ at each sub-center laboratory), with a body mass index (BMI) of $19-30 \mathrm{~kg} / \mathrm{m}^{2}$. Participants were excluded if they had participated in any other clinical trial within the prior 3 months. Individuals were also excluded if they met one or more of the following criteria: (1) had type 1 diabetes mellitus; (2) were diabetic and had previously treated or untreated FPG $\geq 8 \mathrm{mmol} /$ $\mathrm{L}$ or 2 -hr PPG $\geq 17 \mathrm{mmol} / \mathrm{L}$; (3) were women of childbearing age who were pregnant, breastfeeding, or intended to become pregnant or were not using adequate contraceptive methods; (4) were allergic to the study drugs; (5) were unable to cooperate; (6) had impaired liver function, defined as an aspartate aminotransferase or alanine transaminase more than twice the upper limit of normal; (7) had impaired renal function, defined as serum creatinine $\geq 133 \mu \mathrm{mol} / \mathrm{L}$; (8) had uncontrolled treated/untreated severe hypertension (systolic blood pressure $\geq 160 \mathrm{mmHg}$ and/or diastolic blood pressure $\geq 95 \mathrm{mmHg}$ ); (9) had any chronic gastrointestinal disease (pancreatitis, inflammatory bowel disease) or history of intestinal surgery; (10) had severe heart disease, such as heart failure, unstable angina pectoris, acute myocardial infarction; (11) had chronic hypoxic diseases such as emphysema or pulmonary heart disease; (12) had obvious diseases of the blood system; (13) had tumor diseases or endocrine diseases, such as hyperthyroidism or hypercortisolism; (14) had mental illness or had abused alcohol, drugs, or other substances; (15) had received long-term oral or intravenous glucocorticoids hormone therapy; or (16) had stress conditions such as surgery or severe trauma.

\section{Randomization and masking}

The biostatistician, who did not participate in the enrolment of participants, used the statistical software SAS 8.2 PROC PLAN to generate a random code list for 300 participants to receive specific treatments. The stratified, blocked randomization method was used at each center. The test and placebo drugs were provided by the coordinating center in identical internal and external packaging. All of the randomized grouping number segments were sent to the research centers with the corresponding treatment drugs. The study was a double-blind, dualsimulation trial. Participants, investigators, and individuals involved in the analysis of trial data were masked to treatment assignments. A two-level blinding design was used, with the first level being by group (groups A-D) according to the case number, and the second level by treatment (berberine, Bifidobacterium, combination, and placebo).

\section{Procedures}

The study included a run-in period of 2 weeks, in which diabetes education and lifestyle intervention were conducted, and a treatment period of 16 weeks. At the end of the run-in period, 300 eligible subjects were randomly assigned to the following four groups in a ratio of 1:2:1:2 for 16 weeks of treatment: berberine (Be), Bifidobacterium (Bi), berberine and Bifidobacterium (BB), and placebo group. The Be group received oral berberine tablets, $0.5 \mathrm{~g}$, twice a day, and Bifidobacterium placebo capsules, twice a day. The Bi group received Bifidobacterium viable capsules (including $10^{8}$ Bifidobacterium adolescentis), $0.70 \mathrm{~g}$, twice a day, and berberine placebo tablets, twice a day. The BB group received Bifidobacterium viable capsules, $0.70 \mathrm{~g}$, twice a day, and berberine tablets, $0.5 \mathrm{~g}$, twice a day. The placebo group received Bifidobacterium placebo capsules twice a day, and berberine placebo tablets, twice a day. The preparations used were berberine tablets (Northeast Pharmaceutical Group Shenyang First Pharmaceutical Co., Ltd., Lot No.: 130917), berberine tablet simulants with the main component of starch (Northeast Pharmaceutical Group Shenyang First Pharmaceutical Co., Ltd., Lot No.: 20150101), Bifidobacterium viable capsules (Livzon Pharmaceutical Group Inc., Lot No.: 20141013), and Bifidobacterium viable capsules simulants with the main components of lactose and magnesium stearate (Livzon Pharmaceutical Group Inc., Lot No.: 141001). The appearances and characteristics of simulants were similar to berberine tablets and Bifidobacterium viable capsules, respectively.

All of the participants returned to the center every 4 weeks for planned visits during the double-blind intervention periods, and the remaining pills or strips were taken back upon completion of the study and counted to assess adherence. Researchers emphasized the importance of diet and exercise to subjects at each treatment visit. The telephone interview was conducted 2 weeks after the first administration of the study drug to record the medication status and adverse reactions of every participant. The time course for participant registration, intervention, assessment, and follow-up is shown in the Standard Protocol Items: Recommendations for Interventional Trials (see Additional file 1: Table S1). 


\section{Outcomes}

The primary efficacy endpoint was the absolute value of FPG compared with baseline after 16 weeks of treatment in all of the participants, which were assessed by each center. The secondary efficacy endpoints were the changes compared with baseline in (1) 2-hr PPG; (2) glycosylated hemoglobin (HbA1c); (3) blood pressure; (4) lipid metabolism, including total cholesterol (TC), lowdensity lipoprotein cholesterol (LDL-C), high-density lipoprotein cholesterol (HDL-C), and triglycerides (TG); (5) body weight (BW) and BMI; (6) homeostasis model assessment (HOMA) index and insulin early-phase and late-phase secretion index; (7) intestinal glucagon-like peptide-1 (GLP-1); and (8) gut microbiota.

Safety assessments were based on monitoring the vital signs, BW and physical examination, laboratory data, 12lead electrocardiogram, hypoglycemia, and other adverse events.

\section{Data collection}

The methods for the collection of most endpoint measurements were shown in published study protocol [26], except for the gut microbiota.

Feces samples freshly collected from each participant were immediately frozen at $-20^{\circ} \mathrm{C}$, transported to the laboratory in an ice pack and stored at $-80{ }^{\circ} \mathrm{C}$ upon arrival. Bacterial DNA was extracted at Novogene Bioinformatics Technology Co., Ltd. using a Tiangen kit according to the manufacturer's instructions. All samples were paired-end sequenced on the Illumina NovaSeq 6000 (insert size $350 \mathrm{bp}$, read length $151 \mathrm{bp}$ ) at the Novogene Bioinformatics Technology Co., Ltd.

\section{Sequence analysis}

Adaptor and low-quality reads were discarded from the raw reads, and the remaining reads were filtered in order to eliminate human host DNA based on the human genome reference (hg19). Taxonomic profiling of the metagenomic samples was performed using MetaPhlAn2 [27], which uses a library of clade-specific markers to provide pan-microbial quantification at the species level. To obtain the functional profile, the highquality reads were aligned to the updated gut microbiome gene catalog using SOAP2 (v2.22) with a threshold of more than $90 \%$ identity over $95 \%$ of the length. Sequence-based gene abundance profiling was performed as previously described [28]. Pathway enrichment analyses are based on KEGG annotation and the reporter score analysis [29].

\section{Statistical analysis}

This was a pilot study of oral Bifidobacterium. The sample size was estimated using the NCSS PASS 11 (NCSS LLC, Kaysville, UT, USA) software. The sample size calculation was modeled after a previously described method [26]. Based on the decrease of the primary efficacy endpoint of FPG and the results of other studies, the final sample size for each group was determined to be 50 for the Be group, 100 for the Bi group, 50 for the $\mathrm{BB}$ group, and 100 for the placebo group, for a total sample size of 300 .

The intention-to-treat analysis for efficacy endpoints included participants who received at least one dose of the trial drug and had at least one post-treatment data point, comprising the full analysis set. The per-protocol set included participants who adhered adequately to the assigned regimen including undergoing the trial drug treatment according to the protocol without any significant protocol deviation and completing all the evaluations of this study. The safety analyses were based on the safety set, which contained participants who received at least one dose of the trial drug and had at least one safety assessment. The study did not incorporate a planned midpoint analysis.

Efficacy analyses were performed based on the full analysis set. Changes in the primary and secondary efficacy endpoints were assessed using an analysis of covariance model that included terms for grouping, study center, and baseline value. The least squares (LS) mean and corresponding 95\% confidence intervals (CIs) were presented for the changes in each group, and Dunnett LS mean differences $(95 \% \mathrm{CI})$ were provided for the differences between the groups. Differences were considered to be statistically significant when the 95\% CI did not include 0 . Unless otherwise specified, the method of the last observation carried forward was used for efficacy analysis with missing values. Safety assessments were analyzed and adverse events aggregated. The continuous variables or frequency counts and percentages in safety and tolerability data were analyzed using descriptive statistics. The relative indices for hypoglycemic events and changes in weight were compared between the groups according to general principles.

All of the data were analyzed using the SAS 9.3 (SAS Institute Inc., Cary, NC, USA) software with preprogrammed algorithms. Quantitative indices recorded included mean \pm standard deviation and median; qualitative or grade indices were recorded using a frequency distribution table. Two-sided tests were used in all cases, and $P<0.05$ was considered to be statistically significant. Fisher's exact probability test was used to compare the attrition rates between the groups.

Statistical analyses of the gut microbiota were made using the $\mathrm{R}$ software. The differential abundance of phyla, genera, and species was tested with the two-tailed Wilcoxon rank-sum tests, and $P<0.05$ was considered to be statistically significant. All data were not corrected for multiple testing. 


\section{Results}

\section{Participant characteristics}

Between October 2015 and April 2018, 515 participants were screened. Three hundred of these participants were deemed to be eligible and completed randomization (Fig. 1). The intention-to-treat analysis was performed for a total of 297 participants of the full analysis set (Be group $n=49$ participants, Bi group $n=100$ participants, BB group $n=49$ participants, and placebo group $n=99$ participants). The per-protocol set included 245 participants. The safety set included 297 participants. Baseline demographic and clinical characteristics did not differ among the groups (Table 1).

\section{Changes in primary and secondary efficacy endpoints}

The changes in the primary and secondary efficacy endpoints between baseline and after 16 weeks of treatment are shown in Table 2. The primary efficacy endpoint, FPG, changed from $6.49 \pm 0.64$ at baseline to $6.20 \pm$ $0.80 \mathrm{mmol} / \mathrm{L}$ at 16 weeks in the Be group, from $6.40 \pm$ 0.66 to $6.43 \pm 0.93 \mathrm{mmol} / \mathrm{L}$ in the Bi group, from $6.42 \pm$ 0.77 to $6.16 \pm 0.91 \mathrm{mmol} / \mathrm{L}$ in the $\mathrm{BB}$ group, and from $6.43 \pm 0.71$ to $6.67 \pm 1.34 \mathrm{mmol} / \mathrm{L}$ in the placebo group.
Compared with the placebo group, a pronounced effect of lowering FPG was observed in the Be and BB groups with an LS mean difference of $-0.50,95 \%$ CI $[-0.85$, - 0.15] $\mathrm{mmol} / \mathrm{L}$ and $-0.55,95 \% \mathrm{CI}[-0.91,-0.20] \mathrm{mmol} /$ $\mathrm{L}$, respectively. However, there was no significant difference between the $\mathrm{Be}$ and $\mathrm{BB}$ groups with an LS mean difference of $-0.05,95 \%$ CI $[-0.47,0.36] \mathrm{mmol} / \mathrm{L}$. In addition, reduction of FPG was not shown in the Bi group (LS mean difference of $-0.19,95 \%$ CI $[-0.47,0.09$ ] $\mathrm{mmol} / \mathrm{L})$.

We also found similar improvements of 2-hr PPG to FPG. Compared with the placebo group, more reductions of 2-hr PPG were observed in the Be and BB groups with an LS mean difference of $-1.37,95 \% \mathrm{CI}$ $[-2.42,-0.32] \mathrm{mmol} / \mathrm{L}$ and $-1.59,95 \% \mathrm{CI}[-2.62,-0.55]$ $\mathrm{mmol} / \mathrm{L}$, respectively. Only the BB group showed a significant reduction of HbA1c compared with the placebo group with an LS mean difference of -0.23 , 95\% CI $[-0.38,-0.07] \%$, instead of the Be group (LS mean difference of $-0.06,95 \%$ CI $[-0.21,0.10] \%$ ) (Table 2), which may indicate that a better hypoglycemic effect was observed in the BB group. There were significant differences in lipid levels among the groups, including TC, LDL-C,

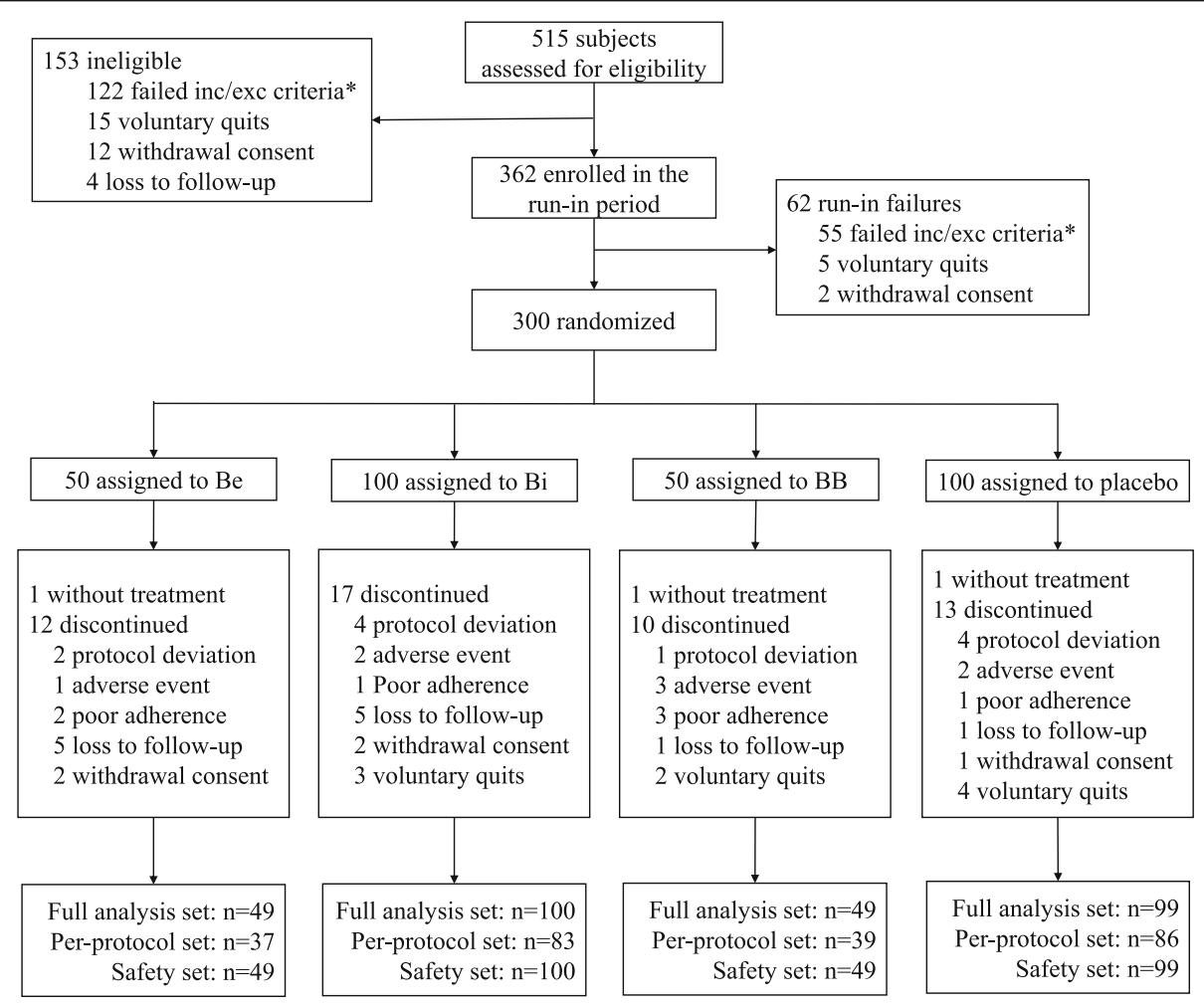

Fig. 1 Trial profile. *The criteria were not mutually exclusive. The full analysis set, as the primary analysis set for this study, included participants who received at least one dose of the trial drug and had at least one post-treatment data point. The per-protocol set included participants who adhered adequately to the assigned regimen, including undergoing the trial drug treatment according to the protocol without any significant protocol deviation and completing all the evaluations of this study. The safety set included participants who received at least one dose of the trial drug and had at least one safety assessment 
Table 1 Baseline characteristics of subjects (full analysis set)

\begin{tabular}{|c|c|c|c|c|c|}
\hline Variable & $\mathrm{Be}$ & $\mathrm{Bi}$ & BB & Placebo & $P$ value \\
\hline$n(\%)$ & $49(98.00)$ & $100(100.00)$ & $49(98.00)$ & $99(99.00)$ & \\
\hline Age, years & $53.28 \pm 9.87$ & $54.16 \pm 9.10$ & $53.36 \pm 9.49$ & $52.73 \pm 9.35$ & 0.761 \\
\hline Sex (male), $n(\%)$ & $23(46.94)$ & $59(59.00)$ & $27(55.10)$ & $54(54.55)$ & 0.585 \\
\hline $\mathrm{BW}, \mathrm{kg}$ & $68.37 \pm 10.82$ & $69.57 \pm 9.06$ & $69.04 \pm 10.48$ & $69.58 \pm 11.24$ & 0.904 \\
\hline $\mathrm{BMl}, \mathrm{kg} / \mathrm{m}^{2}$ & $25.05 \pm 2.68$ & $25.25 \pm 2.28$ & $25.20 \pm 2.69$ & $25.02 \pm 2.80$ & 0.921 \\
\hline Waist circumference, $\mathrm{cm}$ & $90.08 \pm 9.25$ & $90.98 \pm 7.58$ & $91.82 \pm 8.13$ & $91.13 \pm 8.58$ & 0.774 \\
\hline Hip circumference, $\mathrm{cm}$ & $98.17 \pm 5.73$ & $98.72 \pm 7.74$ & $98.60 \pm 6.20$ & $98.10 \pm 6.81$ & 0.920 \\
\hline Systolic blood pressure, $\mathrm{mmHg}$ & $120.56 \pm 11.88$ & $123.08 \pm 12.42$ & $121.17 \pm 13.75$ & $122.71 \pm 11.37$ & 0.591 \\
\hline Diastolic blood pressure, $\mathrm{mmHg}$ & $77.46 \pm 8.94$ & $78.57 \pm 8.19$ & $77.97 \pm 7.82$ & $76.86 \pm 7.68$ & 0.516 \\
\hline Pulse, beats/min & $73.14 \pm 8.29$ & $74.15 \pm 8.56$ & $74.80 \pm 7.20$ & $74.17 \pm 9.18$ & 0.812 \\
\hline ECG & & & & & 0.225 \\
\hline Normal, n (\%) & 39 (79.59) & $64(64.00)$ & $29(59.18)$ & $63(63.64)$ & \\
\hline Non-clinical significance, $n(\%)$ & $9(18.37)$ & $33(33.00)$ & $17(34.69)$ & $26(26.26)$ & \\
\hline Clinical significance, $n(\%)$ & $1(2.04)$ & $3(3.00)$ & $3(6.12)$ & $9(9.09)$ & \\
\hline None, $n(\%)$ & $0(0.00)$ & $0(0.00)$ & $0(0.00)$ & $1(1.01)$ & \\
\hline $\mathrm{FPG}, \mathrm{mmol} / \mathrm{L}$ & $6.49 \pm 0.64$ & $6.40 \pm 0.66$ & $6.42 \pm 0.77$ & $6.43 \pm 0.71$ & 0.881 \\
\hline 30 min post-plasma glucose, $\mathrm{mmol} / \mathrm{L}$ & $12.12 \pm 1.63$ & $11.93 \pm 1.84$ & $11.76 \pm 2.09$ & $11.94 \pm 1.86$ & 0.830 \\
\hline 2-hr PPG, mmol/L & $11.17 \pm 2.41$ & $11.40 \pm 3.08$ & $11.24 \pm 2.37$ & $11.62 \pm 3.08$ & 0.906 \\
\hline $\mathrm{HbA} 1 \mathrm{c}, \%$ & $6.19 \pm 0.53$ & $6.19 \pm 0.59$ & $6.12 \pm 0.72$ & $6.23 \pm 0.68$ & 0.920 \\
\hline Fasting serum insulin, $\mu \mathrm{IU} / \mathrm{mL}$ & $9.87(5.91-13.60)$ & $10.52(7.65-13.88)$ & $9.56(7.49-13.89)$ & $9.78(6.69-11.98)$ & 0.430 \\
\hline 30 min post-serum insulin, $\mu \mathrm{lU} / \mathrm{mL}$ & $39.16(21.46-64.74)$ & $33.00(19.75-61.83)$ & $34.30(20.40-51.74)$ & $32.98(23.88-52.84)$ & 0.962 \\
\hline 2-hr post-serum insulin, $\mu \mathrm{IU} / \mathrm{mL}$ & $61.85(40.88-94.43)$ & $58.56(40.50-84.36)$ & $69.15(46.19-88.76)$ & $56.26(36.35-79.12)$ & 0.279 \\
\hline Fasting serum $C$ peptide, $n g / m L$ & $1.46(0.60-2.04)$ & $1.58(0.60-2.28)$ & $1.38(0.72-2.13)$ & $1.47(0.63-2.13)$ & 0.995 \\
\hline 30 min post-serum C peptide, $\mathrm{ng} / \mathrm{mL}$ & $3.29(2.07-4.44)$ & $2.67(1.52-3.87)$ & $2.76(1.77-3.84)$ & $2.79(1.08-4.53)$ & 0.711 \\
\hline 2-hr post-serum C peptide, $\mathrm{ng} / \mathrm{mL}$ & $6.01(3.09-8.92)$ & $5.64(2.16-8.74)$ & $6.76(3.39-8.35)$ & $5.82(2.40-8.32)$ & 0.745 \\
\hline $\mathrm{TG}, \mathrm{mmol} / \mathrm{L}$ & $2.12(1.50-2.61)$ & $1.71(1.21-2.46)$ & $1.66(1.03-2.14)$ & $1.83(1.20-2.35)$ & 0.253 \\
\hline $\mathrm{TC}, \mathrm{mmol} / \mathrm{L}$ & $5.03 \pm 1.15$ & $4.76 \pm 1.01$ & $4.44 \pm 0.96$ & $4.72 \pm 0.88$ & 0.035 \\
\hline $\mathrm{HDL}-\mathrm{C}, \mathrm{mmol} / \mathrm{L}$ & $1.15 \pm 0.30$ & $1.19 \pm 0.31$ & $1.12 \pm 0.27$ & $1.15 \pm 0.26$ & 0.535 \\
\hline $\mathrm{LDL}-\mathrm{C}, \mathrm{mmol} / \mathrm{L}$ & $3.00 \pm 0.88$ & $2.85 \pm 0.80$ & $2.67 \pm 0.84$ & $2.81 \pm 0.79$ & 0.260 \\
\hline HOMA-IR & $2.69(1.76-3.41)$ & $3.05(1.88-4.08)$ & $2.74(2.16-3.95)$ & $2.75(1.89-3.45)$ & 0.450 \\
\hline HOMA- $\beta$ & $69.66(42.97-98.37)$ & $71.98(53.23-105.48)$ & $68.00(46.41-96.77)$ & $63.53(48.40-87.79)$ & 0.832 \\
\hline Fasting GLP-1, pmol/L & $2.38(1.64-5.24)$ & $2.00(1.53-5.11)$ & $2.95(1.52-5.82)$ & $2.71(1.66-5.99)$ & 0.895 \\
\hline 30 min post-GLP-1, pmol/L & $4.39(2.71-7.03)$ & $4.62(2.62-7.29)$ & $3.77(2.62-7.28)$ & $3.98(2.37-6.97)$ & 0.982 \\
\hline 2-hr post-GLP-1, pmol/L & $2.31(1.24-4.34)$ & $2.48(1.80-5.04)$ & $3.32(1.92-5.32)$ & $2.48(1.40-5.04)$ & 0.745 \\
\hline Area under the curve of GLP-1 & $7.48(4.53-14.40)$ & $8.13(4.91-12.90)$ & $6.99(5.31-13.69)$ & $6.95(4.72-13.34)$ & 0.905 \\
\hline
\end{tabular}

Data were presented as $n(\%)$, mean \pm standard deviation, and median (range interquartile). $P$ value: comparison among the groups after treatment ECG explanation: normal, normal ECG; non-clinical significance, abnormal ECG without any clinical significance; clinical significance, abnormal ECG with clinical significance; none, ECG was not performed

HOMA-IR: (fasting serum insulin $\times$ fasting plasma glucose)/22.5, homeostasis model assessment index for assessing insulin resistance; HOMA- $\beta$ : (20 $\times$ fasting serum insulin)/(fasting plasma glucose - 3.5), homeostasis model assessment index for assessing $\beta$ cell function. $P$ value: comparison among the groups; Be: treatment with berberine; Bi: treatment with Bifidobacterium; BB: treatment with berberine and Bifidobacterium

$B W$ body weight, $B M I$ body mass index, FPG fasting plasma glucose, 2-hr PPG 2-hour postprandial plasma glucose, $T G$ triglyceride, $T C$ total cholesterol, HDL-C highdensity lipoprotein cholesterol, LDL-C low-density lipoprotein cholesterol, ECG electrocardiogram

and HDL-C. The similar effect of lowering TC level was shown in the Be group and BB group when compared with the placebo group, with an LS mean difference of $-0.31,95 \% \mathrm{CI}[-0.59,-0.04] \mathrm{mmol} / \mathrm{L}$ and -0.44 ,
95\% CI $[-0.72,-0.16] \mathrm{mmol} / \mathrm{L}$, respectively. The changes of $\mathrm{TC}$ level in the $\mathrm{Bi}$ group were similar to the placebo group (LS mean difference of $-0.04,95 \%$ CI $[-0.26,0.18] \mathrm{mmol} / \mathrm{L})$. Only the BB group showed 


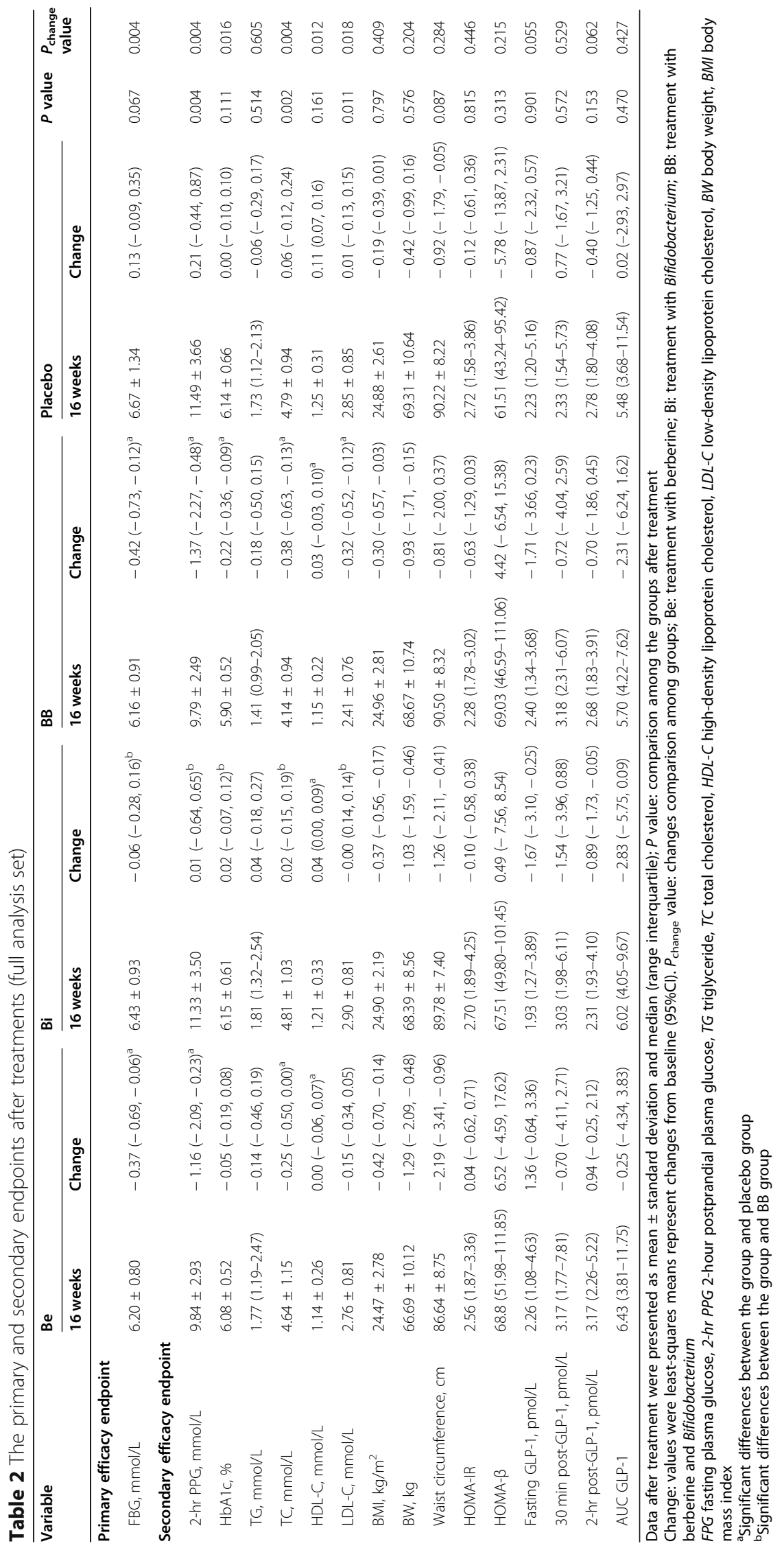


that LDL-C was significantly decreased compared with the placebo group, with an LS mean difference of -0.32 , 95\% CI $[-0.55,-0.10] \mathrm{mmol} / \mathrm{L}$. The HDL-C levels were slightly increased in the $\mathrm{Be}, \mathrm{Bi}$, and $\mathrm{BB}$ groups compared with the placebo group, with an LS mean difference of $-0.11,95 \%$ CI $[-0.18,-0.03] \mathrm{mmol} / \mathrm{L},-0.07$, 95\% CI $[-0.13,-0.01] \mathrm{mmol} / \mathrm{L}$, and $-0.08,95 \% \mathrm{CI}$ $[-0.15,-0.01]$, respectively. Additionally, there were no significant differences in other metabolic-related indicators among the groups.

We conducted sensitivity analyses based on perprotocol set, which showed similar results with those based on full analysis set (see Additional file 2: Table S2).

\section{Comparison of composition and function of gut microbiota}

To characterize the gut microbiota of all groups, 208 high-quality, paired fecal samples collected at baseline and 16 weeks (Be group, $n=30$; Bi group, $n=62$; BB group, $n=30$; and placebo group, $n=86$ ) were performed with whole metagenomics sequencing. Next, we compared the demographic and clinical characteristics between participants with and without fecal samples, but there was no significant difference between them (see Additional file 3: Table S3). At the community level, more changes in the structure of the gut microbiota were observed in the Be and $\mathrm{BB}$ groups after treatment. The gene richness of the Be group decreased significantly compared with baseline (see Additional file 4: Fig. S1A, $P=0.009$ ), and the $\mathrm{BB}$ group also showed decreased tendency (see Additional file 4: Fig. S1A, $P=$ 0.055). The Bray-Curtis distance showed that gut microbiota changes between baseline and after treatment in the $\mathrm{Be}$ and $\mathrm{BB}$ groups were significantly larger than those in the $\mathrm{Bi}$ and placebo groups (see Additional file 4: Fig. S1B). There were no significant differences in gene richness or Bray-Curtis distance between the $\mathrm{Bi}$ and placebo groups.

After filtering out species with a low occurrence (i.e., present in fewer than $30 \%$ of individuals), we found that the abundance of Firmicutes was slightly lowered in the Be group, but higher in the $\mathrm{BB}$ group. Consistent changes were also observed in the Be group and $\mathrm{BB}$ group, including decreased abundance of Roseburia and increased abundance of Blautia including Ruminococcus gnavus and Ruminococcus torques. The abundance of Actinobacteria was increased only in the Bi group (Fig. 2).

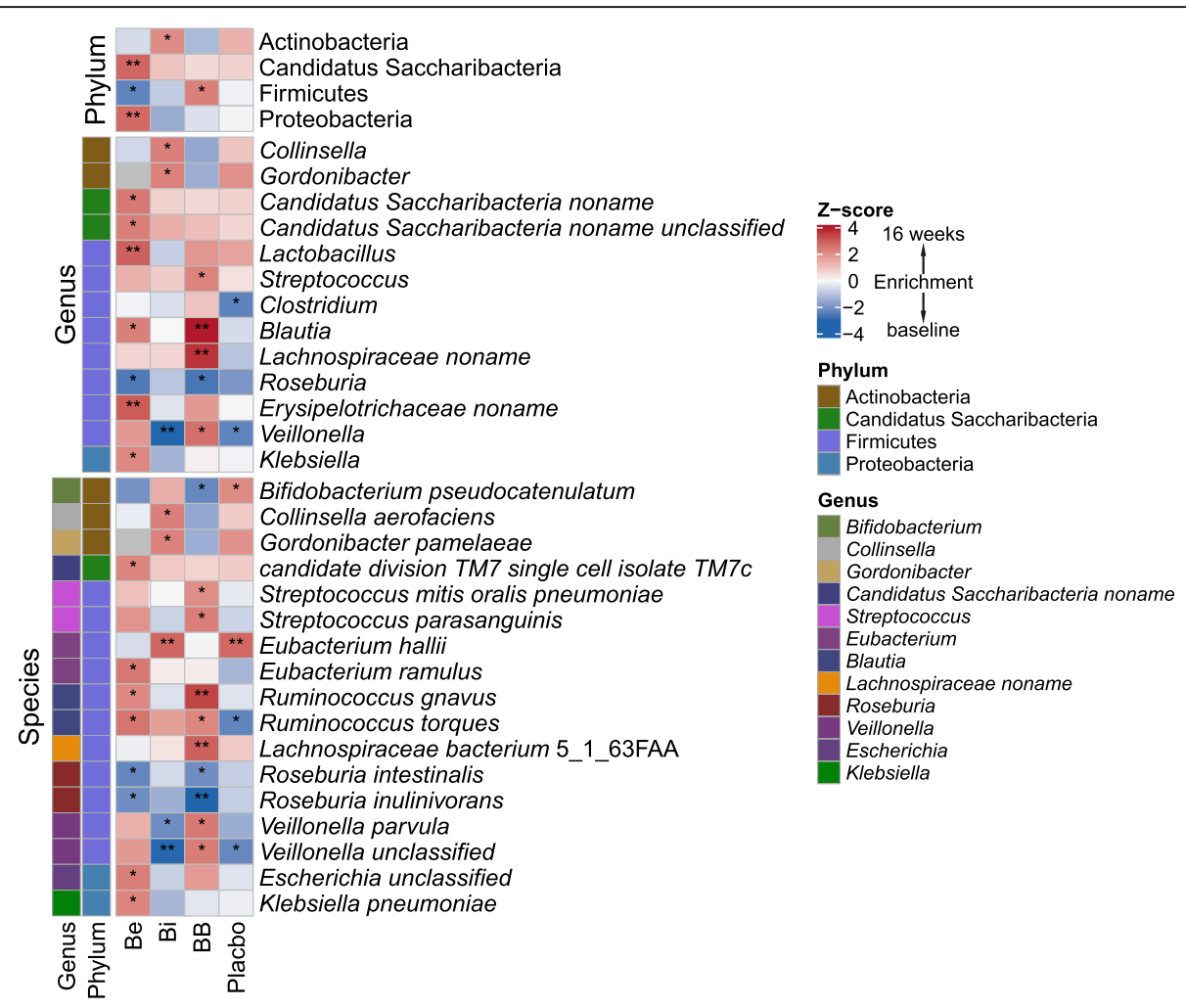

Fig. 2 Heat map showing differentially abundant taxa of the fecal microbiota between baseline and 16 weeks of treatment in four experiments. The values of color in the heat map represent the $Z$-score. Only bacterial taxa that were significant in one of the experiments were included. The Wilcoxon matched-pairs signed-rank tests were used, ${ }^{*} P<0.05 ;{ }^{*} P<0.01$ 
Furthermore, we found that the abundance of Proteobacteria was significantly higher in the Be group, including the opportunistic pathogen Klebsiella pneumoniae. However, this phenomenon was not observed in the $\mathrm{BB}$ group, which may be attributable to the use of Bifidobacterium.

According to further analysis about functional characterization, we found that some carbohydrate and lipid metabolism pathways, including galactose metabolism (map00052), fructose and mannose metabolism (map00051), and glycerolipid metabolism (map00561) pathways, were enriched in the Be and BB groups, which may mainly result from the use of berberine (see Additional file 5: Table S4). The Be group was characterized by enriched pathways of xenobiotic biodegradation and metabolism associated with harmful chemical products, including toluene degradation (map00623), benzoate degradation (map00362), fluorobenzoate degradation (map00364), and chlorocyclohexane and chlorobenzene degradation (map00361), but these changes were inverse in Bi group (see Additional file 5: Table S4). Whether this phenomenon was another benefit of Bifidobacterium needs to be further assessed.

\section{Safety assessments}

There were no significant differences among the groups in the incidence of adverse events, any drug-related adverse events, any adverse events leading to discontinuation, and severe adverse events. Hypoglycemia occurred seven times in seven participants of the BB group, 14 times in nine participants of the Be group, 23 times in 14 participants of the Bi group, and 19 times in 14 participants of the placebo group. There were also no significant differences in the incidence of hypoglycemia among the groups (Table 3).

\section{Discussion}

This study has shown that FPG were decreased significantly after 16 weeks of treatment in both the Be and BB groups. However, lowering 2-hr PPG showed a stronger effect in the BB group. No obvious hypoglycemic effect was observed in the Bi group. We also characterized the gut microbiota of all the groups and found that berberine could regulate the gut microbiota in human hyperglycemia, which provided some evidence for the intestinal mechanism underlying berberine's hypoglycemic effect. To the best of our knowledge, this was the first

Table 3 Summary of adverse events (safety set)

\begin{tabular}{|c|c|c|c|c|c|c|c|c|c|}
\hline \multirow[t]{2}{*}{ Adverse events } & \multicolumn{2}{|c|}{$\mathrm{Be}(n=49)$} & \multicolumn{2}{|c|}{$\mathrm{Bi}(n=100)$} & \multicolumn{2}{|c|}{$\mathrm{BB}(n=49)$} & \multicolumn{2}{|c|}{ Placebo $(n=99)$} & \multirow[t]{2}{*}{$P$ value } \\
\hline & $\begin{array}{l}\text { Events, } \\
n\end{array}$ & $\begin{array}{l}\text { Participant, } \\
n(\%)\end{array}$ & $\begin{array}{l}\text { Events, } \\
n\end{array}$ & $\begin{array}{l}\text { Participant, } \\
n(\%)\end{array}$ & $\begin{array}{l}\text { Events, } \\
n\end{array}$ & $\begin{array}{l}\text { Participant, } \\
n(\%)\end{array}$ & $\begin{array}{l}\text { Events, } \\
n\end{array}$ & $\begin{array}{l}\text { Participant, } \\
n(\%)\end{array}$ & \\
\hline Adverse events & 111 & $40(81.63)$ & 203 & $84(84.00)$ & 118 & $40(81.63)$ & 239 & $82(82.83)$ & 0.973 \\
\hline Drug-related adverse events & 19 & $9(18.37)$ & 30 & $21(21.00)$ & 28 & $15(30.61)$ & 37 & $26(26.26)$ & 0.430 \\
\hline $\begin{array}{l}\text { Adverse events unrelated to the } \\
\text { study drug }\end{array}$ & 92 & $38(77.55)$ & 173 & $81(81.00)$ & 90 & $37(75.51)$ & 202 & $79(79.80)$ & 0.859 \\
\hline $\begin{array}{l}\text { Adverse events leading to } \\
\text { discontinuation }\end{array}$ & 4 & $2(4.08)$ & 4 & $4(4.00)$ & 0 & $0(0.00)$ & 6 & $3(3.03)$ & 0.679 \\
\hline Severe adverse events & 5 & $4(8.16)$ & 4 & $4(4.00)$ & 0 & $0(0.00)$ & 6 & $3(3.03)$ & 0.213 \\
\hline Hypoglycemia event & 14 & $9(18.37)$ & 23 & $14(14.00)$ & 7 & $7(14.29)$ & 19 & $14(14.14)$ & 0.887 \\
\hline \multicolumn{10}{|l|}{$\begin{array}{l}\text { Most frequent treat-related adverse } \\
\text { events }\end{array}$} \\
\hline Fecal abnormalities & 5 & $3(6.12)$ & 12 & $8(8.00)$ & 7 & $6(12.24)$ & 12 & $9(9.09)$ & \\
\hline $\begin{array}{l}\text { Abdominal discomfort/digestive } \\
\text { tract disease }\end{array}$ & 9 & $5(10.20)$ & 7 & $6(6.00)$ & 14 & $9(18.37)$ & 8 & $7(7.07)$ & \\
\hline Dental and oral disorders & 0 & $0(0.00)$ & 0 & $0(0.00)$ & 1 & $1(2.04)$ & 1 & $1(1.01)$ & \\
\hline Others & 1 & $1(2.04)$ & 1 & $1(1.00)$ & 0 & $0(0.00)$ & 0 & $0(0.00)$ & \\
\hline Upper respiratory tract infection & 0 & $0(0.00)$ & 0 & $0(0.00)$ & 2 & $2(4.08)$ & 1 & $1(1.01)$ & \\
\hline Changes in body weight & 0 & $0(0.00)$ & 3 & $3(3.00)$ & 1 & $1(2.04)$ & 1 & $1(1.01)$ & \\
\hline Dizziness & 0 & $0(0.00)$ & 1 & $1(1.00)$ & 2 & $2(4.08)$ & 1 & $1(1.01)$ & \\
\hline Trauma/arthropathy & 0 & $0(0.00)$ & 1 & $1(1.00)$ & 0 & $0(0.00)$ & 0 & $0(0.00)$ & \\
\hline $\begin{array}{l}\text { Abnormal ECG/cardiac } \\
\text { dysfunction }\end{array}$ & 3 & $3(6.12)$ & 1 & $1(1.00)$ & 1 & $1(2.04)$ & 2 & $2(2.02)$ & \\
\hline $\begin{array}{l}\text { Blood routine/biochemistry/ } \\
\text { urinalysis }\end{array}$ & 1 & $1(2.04)$ & 4 & $3(3.00)$ & 0 & $0(0.00)$ & 10 & $7(7.07)$ & \\
\hline Hemorrhoids & 0 & $0(0.00)$ & 0 & $0(0.00)$ & 0 & $0(0.00)$ & 1 & $1(1.01)$ & \\
\hline
\end{tabular}


multi-center, randomized, parallel-controlled study to report the hypoglycemic effect of berberine and single Bifidobacterium that were used synergistically on patients with hyperglycemia.

As expected, our results showed that berberine (0.5 g, twice a day) had a hypoglycemic effect in patients newly diagnosed with hyperglycemia compared with placebo, especially in reducing FPG and 2-hr PPG. This was consistent with the results from previous clinical trials [30, 31], which indicated that berberine treatment was associated with different degrees of reduction on FPG, 2-hr PPG and HbA1c. However, significant changes in HbA1c were not observed in the Be group. This may be attributed to the relatively low mean glucose level of enrolled participants, or perhaps the treatment period was not long enough. Therefore, this study provided evidence for the hypoglycemic effect of berberine in the relatively early stage of T2D.

Moreover, although more effective lowering of the HbA1c effect was not observed directly in the BB group compared with the Be group (LS mean difference -0.17 , $95 \%$ CI $[-0.35,0.01] \%)$, only the BB group showed a significant reduction of $\mathrm{HbA1c}$ compared with the placebo group (LS mean difference of $-0.23,95 \% \mathrm{CI}$ $[-0.38,-0.07] \%)$, instead of the Be group (LS mean difference of $-0.06,95 \%$ CI $[-0.21,0.10] \%)$. These results indicated that $B$. adolescentis may enhance the hypoglycemic effect of berberine. Supplementation with $B$. adolescentis in a rodent model of the metabolic syndrome has been shown to increase insulin sensitivity [32]. Bifidobacterium viable capsules (B. adolescentis) used in this trial are approved by the Chinese State Food and Drug Administration for clinical use (approval number S10960040) to treat intestinal dysfunction caused by an imbalance of the gut microbiota in clinical practice. The detailed drug instruction can be found on the website (https://www.livzon.com.cn/product/21.html). Patients with T2D were characterized by a moderate degree of gut microbial dysbiosis [33]. Furthermore, a study reported that metformin promoted the growth of $B$. adolescentis both in vivo and in vitro using pure cultures. The study also observed a negative correlation between the peak-to-trough ratio of $B$. adolescentis and HbA1c, which suggested that increased growth of this bacterial species could potentially contribute to the antidiabetic effect of metformin [4]. Metformin and berberine similarly shifted the overall structure of the gut microbiota in rats [34]. Therefore, B. adolescentis may improve the intestinal environment to promote berberine's ability to play a hypoglycemic role. Unfortunately, the hypoglycemic effect of $B$. adolescentis was not observed in our study. Another study also showed that there was not a superior effect of probiotics (multi-strain probiotics, $\geq 50$ billion
CFU) in treating T2D compared to that of placebo or probiotics plus berberine or compared to that of berberine [31]. The types and dosages of probiotics used were also different from this study. We chose the dose of $2 \times 10^{8}$ CFU per day according to the manufacturer's drug instructions for safety reasons. The low dose of probiotics in this study should be acknowledged. The optimal and most clinically relevant probiotic medium, strain, dose, and duration of intervention have not yet been fully described. Data in a review suggested that multi-strain probiotic interventions providing seven million to 100 billion CFU administered for 6 to 12 weeks are efficacious for improving glycemic control in T2D patients [35]. Therefore, whether the different probiotic strains, the number of probiotics ingested, or the patient's race could lead to different hypoglycemic effects are worthy topics of further study.

From animal and in vitro studies, berberine is known to act as an antidiabetic agent through stimulating the glucose uptake of cells, inhibiting gluconeogenesis to reduce hepatic glucose output, increasing the expression of insulin receptors and the secretion of GLP-1, and suppressing the activity of intestinal disaccharidases [36]. A variety of molecular mechanisms for berberine have been proposed, such as AMPK activation, glucose transport stimulation, mitochondrial inhibition, and anti-oxidation [36]. Moreover, berberine could regulate the gut microbiota, increase the production of gut shortchain fatty acids, alter microbial bile acid metabolism and the intestinal farnesoid $\mathrm{X}$ receptor signaling pathway, and affect the synthesis and transport of amino acids [10]. To discern the intestinal mechanism of berberine's hypoglycemic effect, we tested fecal samples of participants and found that the gut microbiota after treatment changed more compared with baseline in the $\mathrm{BB}$ and Be groups. The significant colonization of $B$. adolescentis was not observed after oral supplementation with $B$. adolescentis, yet probiotic gut mucosal colonization efficacy remains controversial [37]. Further study will be needed to decide whether this phenomenon occurs on account of low-dose probiotics or high levels of acid and bile acids in the stomach and duodenum. In addition, significant increases of Blautia were observed in both the $\mathrm{BB}$ and Be groups. $R$. gnavus and $R$. torques belonging to Blautia were consistently enriched in both the $\mathrm{Be}$ and $\mathrm{BB}$ groups compared with baseline. Indeed, $R$. gnavus was significantly correlated with the improvement of glucose homeostasis and insulin sensitivity [38]. $R$. gnavus has been reported to be an ursodeoxycholic acid (UDCA) producer, and its colonization was uniquely associated with the production of UDCA in children [39, 40]. The pharmaceutical benefits of UDCA are well known, as it is commonly administrated for the treatment or prevention of various diseases or symptoms associated with disorders of bile acid metabolism [41]. 
R. gnavus may be involved in intestinal tryptophan metabolism [42] and excretion of the neurotransmitter tryptamine in vitro [43]. It is also reported that $R$. gnavus expresses $\alpha$-galactosidase, which plays essential roles in the metabolism of dietary oligosaccharides [44] and thus may be associated with galactose metabolism enrichment in the BB and Be groups in functional analysis. Therefore, whether $R$. gnavus potentiates the hypoglycemic effect of berberine must be further verified by animal experiments.

However, not all of the changes observed in the regulatory effect of berberine on patients with T2D were beneficial, including a decrease of some probiotics (such as the butyric acid producer Roseburia) and an increase of some opportunistic pathogens (Proteobacteria and Streptococcus); this observation is consistent with other studies [9, 12]. However, the increase of opportunistic pathogen growth caused by berberine could be offset through the supplementation of Bifidobacterium. The species of Klebsiella pneumoniae, Roseburia intestinalis, Roseburia hominis, and R. gnavus were regarded as the key berberine-responding species in another study [31]. They also found that the hypoglycemic effect of berberine was mediated by the gut microbiota, which inhibited the biotransformation of deoxycholic acid [31]. However, it was different from our study in that participants were given an oral broad-spectrum antibiotic for 7 days before berberine treatment [31].

We also observed significant decreases in gene richness in the Be group and mild decreases in the $\mathrm{BB}$ group, which may be correlated to the antibacterial activity by berberine, and decreased gene richness is mildly reversed by Bifidobacterium. Li et al. showed that the concomitant use of prebiotic could slightly reverse the reduced diversity and richness of microbiota caused by berberine and produce better glycometabolism than berberine alone in diabetic mice [45]. Another possible benefit of Bifidobacterium was observed in the function analysis. It could also offset enriched pathways of harmful chemical products by berberine including toluene, benzoate, fluorobenzoate, chlorocyclohexane, and chlorobenzene degradation. We also found some carbohydrate metabolism pathways, which were associated with the production of short-chain fatty acids. These were enriched in groups with berberine including galactose, fructose, and mannose metabolism [46]. Another study also showed that berberine could increase the intestinal short-chain fatty acids content in $\mathrm{db} / \mathrm{db}$ mice [47].

Berberine could affect the secretion of GLP-1 in vivo and in vitro directly or indirectly $[48,49]$. However, the evidence that berberine regulates human GLP-1 secretion is still absent. Although our results also showed that changes in GLP-1 concentration were similar among the groups, one cannot rule out that the lack of change in plasma GLP-1 concentration is not affected by berberine or Bifidobacterium. In fact, GLP-1 is secreted after a meal in the portal vein, where it exerts its physiologic role [50] and is rapidly degraded by the DPP-IV enzyme within $90 \mathrm{~s}$ [51]. Therefore, further study is needed to determine whether berberine increases GLP-1 secretion in humans.

Some changes in gut microbiota composition and functional analysis were also observed in the placebo and $\mathrm{Bi}$ groups. This result could indicate that some functional changes may be related to dietary (and exercise) recommendations in the period. Lifestyle intervention was shown to modify the gut microbiota in metabolic diseases $[52,53]$. We observed that Bifidobacterium pseudocatenulatum and Eubacterium hallii were increased after intervention in the placebo group. E. hallii was also significantly increased in the Bi group. E. hallii was significantly correlated with the improvements of glucose homeostasis and insulin sensitivity [38]. Oral administration of $B$. pseudocatenulatum could reverse the adverse effects of diet-induced obesity through the gut-bone axis [54]. Both the $\mathrm{Bi}$ and placebo groups showed that the pathways of glycosaminoglycan degradation were increased and benzoate degradation was decreased. The functional analysis also showed some beneficial changes, including decreased pathways of $A B C$ transporters and lipopolysaccharide biosynthesis after intervention in the placebo group.

Nonetheless, some limitations of this study should be acknowledged. First, this multi-center trial was conducted in the same geographic area; therefore, the conclusions may not be generalizable to other regions. Whether this phenomenon is prevalent across multiple regions needs further verification. Second, we observed the hypoglycemic effect at 16 weeks, but it is unknown whether prolonged treatment will cause further changes to the gut microbiota and other metabolic indices. Third, the sample size of stool was limited for subgroup analysis. Fourth, detailed dietary records were absent. Finally, further studies in vitro and on animals were not performed to explain the potential mechanism.

\section{Conclusions}

In summary, the hypoglycemic effect of berberine was further validated, and Bifidobacterium showed the potential to enhance the hypoglycemic effect of berberine. We also observed possible changes in the gut microbiota when regulated by berberine for lowering glucose, which provided a basis for the study of its hypoglycemic mechanism and for clinical use of berberine as a safe and effective hypoglycemic drug in human hyperglycemia. Given the benefits of Bifidobacterium and berberine lowering blood glucose, more studies are needed to confirm these findings. 


\section{Abbreviations}

T2D: Type 2 diabetes; FPG: Fasting plasma glucose; 2-hr PPG: 2-hour postprandial plasma glucose; BMI: Body mass index; TC: Total cholesterol; LDL-C: Low-density lipoprotein cholesterol; HDL-C: High-density lipoprotein cholesterol; LS: Least squares; Cl: Confidence interval; UDCA: Ursodeoxycholic acid

\section{Supplementary Information}

The online version contains supplementary material available at https://doi. org/10.1186/s13073-021-00942-7.

Additional file 1: Table S1. Specific research plan and implementation steps.

Additional file 2: Table S2. The primary and secondary endpoints after treatments (per-protocol set).

Additional file 3: Table S3. Comparison of demographic characteristics between subjects with and without fecal samples.

Additional file 4: Figure S1. Differences in four groups on microbial community diversity between baseline and 16 weeks.

Additional file 5: Table S4. Reporter score of the KEGG pathways for comparisons between baseline and treatment.

\section{Acknowledgements}

We thank the Excellence Future International Consulting Co., Ltd. as the contract research organization supervising during data collection and management. We are also grateful to the Northeast Pharmaceutical Group Shenyang First Pharmaceutical Co., Ltd. and Livzon Pharmaceutical Group Inc. for their generosity in providing these drugs.

We particularly thank all of the physicians and participants of the study for their generous cooperation and participation. The authors thank LetPub (www.letpub.com) for its linguistic assistance during the preparation of this manuscript.

\section{Authors' contributions}

QJ, JM, XW, and JZ conceived and designed the study. XX, SL, WY, SL, QH, $Y D, Z T, X G, K M$, and $Y F$ contributed to the data collection. $X Y, X X, W L, C D$, $Z W$, and $X W$ contributed to the data extraction and interpreted the results. $C L, X X$, and $Z W$ performed the data analysis. $J M, X Y$, and $X X$ wrote the first draft. All authors read and approved the final manuscript.

\section{Funding}

This study was funded by the Natural Science Foundation of Shaanxi Province, China (Grant No. 2013KTZB03-02-01); the Key Research and Development Program of Shaanxi Province, China (No. 2017ZDCXLSF0201); and the National Key R\&D Program: Multi-factorial Integrative Management of Type 2 Diabetes (MiDiab) [No. 2017YFC1309803, 2017YFC1309804].

\section{Availability of data and materials}

Sequence data for all microbiome samples are available in the European Nucleotide Archive repository with the accession number PRJEB44907 (https://www.ebi.ac.uk/ena/browser/view/PRJEB44907) [55]. The demographic and clinical dataset of the trial is not available due to this is not compliant with the ethical approval obtained for the study, but can be obtained from the corresponding authors upon reasonable request.

\section{Declarations}

\section{Ethics approval and consent to participate}

This study was approved by the independent Ethics Committee of Xijing Hospital, First Affiliated Hospital of the Fourth Military Medical University (ID: KY20150611-3). Each subject provided signed and dated informed consent before conducting any procedure associated with the study. And this study conformed to the principles of the Declaration of Helsinki.

\section{Consent for publication}

Not applicable.

\section{Competing interests}

The Northeast Pharmaceutical Group Shenyang First Pharmaceutical Co., Ltd. and Livzon Pharmaceutical Group Inc. provided drugs for free. XX, ZW, and $\mathrm{XW}$ are affiliated to Aimigene Institute, which is the research department of Shenzhen 01 Life Science and Technology Ltd. The remaining authors declare that they have no competing interests.

\section{Author details}

${ }^{1}$ Endocrinology Research Center, Department of Endocrinology and Metabolism, Xijing Hospital, Fourth Military Medical University, Xi'an 710032, China. ${ }^{2}$ Aimigene Institute, Shenzhen 518063, China. ${ }^{3}$ Department of General Surgery, Nanjing Drum Tower Hospital, Medical School of Nanjing University, Nanjing, China. ${ }^{4}$ Department of Endocrinology, The Second Affiliated Hospital of Xi'an Jiaotong University, Xi'an, China. ${ }^{5}$ Department of Endocrinology, Affiliated Hospital of Yan'an University, Yan'an, China. ${ }^{6} T$ The Fifth Department of Internal Medicine, Shaanxi Aerospace Hospital, Xi'an, China. ${ }^{7}$ Department of Endocrinology, Xi'an Daxing Hospital, Xi'an, China. ${ }^{8}$ Genertec Universal Xi'an Aero-Engine Hospital, Xi'an, China. ${ }^{9}$ Department of Endocrinology, Xi'an High-Tech Hospital, Xi'an, China. ${ }^{10}$ Department of Endocrinology, Chang'an Hospital, Xi'an, China. ${ }^{11}$ Department of Endocrinology, Xi'an Central Hospital, Xi'an, China. ${ }^{12}$ Department of Endocrinology, Yan'an People's Hospital, Yan'an, China. ${ }^{13}$ Department of Endocrinology, Shangluo Central Hospital, Shangluo, China. ${ }^{14}$ Department of Health Statistics, Fourth Military Medical University, Xi'an, China.

${ }^{15}$ Department of Endocrinology and Metabolism, Shandong Provincial Hospital Affiliated to Shandong University, Jinan 250021, China.

Received: 3 June 2020 Accepted: 21 July 2021

Published online: 09 August 2021

\section{References}

1. Saeedi P, Petersohn I, Salpea P, Malanda B, Karuranga S, Unwin N, et al. Global and regional diabetes prevalence estimates for 2019 and projections for 2030 and 2045: results from the International Diabetes Federation Diabetes Atlas, 9(th) edition. Diabetes Res Clin Pract. 2019:157:107843.

2. American Diabetes A. Pharmacologic approaches to glycemic treatment: standards of medical care in diabetes-2020. Diabetes Care. 2020;43(Suppl 1): S98-S110. https://doi.org/10.2337/dc20-S009.

3. Gu Y, Wang X, Li J, Zhang Y, Zhong H, Liu R, et al. Analyses of gut microbiota and plasma bile acids enable stratification of patients for antidiabetic treatment. Nat Commun. 2017;8(1):1785. https://doi.org/10.103 8/s41467-017-01682-2

4. Wu H, Esteve E, Tremaroli V, Khan MT, Caesar R, Manneras-Holm L, et al. Metformin alters the gut microbiome of individuals with treatment-naive type 2 diabetes, contributing to the therapeutic effects of the drug. Nat Med. 2017:23(7):850-8. https://doi.org/10.1038/nm.4345.

5. Yu M, Jin X, Liang C, Bu F, Pan D, He Q, et al. Berberine for diarrhea in children and adults: a systematic review and meta-analysis. Therap Adv Gastroenterol. 2020;13:1756284820961299.

6. Lan J, Zhao Y, Dong F, Yan Z, Zheng W, Fan J, et al. Meta-analysis of the effect and safety of berberine in the treatment of type 2 diabetes mellitus, hyperlipemia and hypertension. J Ethnopharmacol. 2015;161:69-81. https:// doi.org/10.1016/j.jep.2014.09.049.

7. Zhang Y, Cui YL, Gao LN, Jiang HL. Effects of beta-cyclodextrin on the intestinal absorption of berberine hydrochloride, a P-glycoprotein substrate. Int J Biol Macromol. 2013;59:363-71. https://doi.org/10.1016/j.ijbiomac.2013. 04.074 .

8. Zhang Y, Li X, Zou D, Liu W, Yang J, Zhu N, et al. Treatment of type 2 diabetes and dyslipidemia with the natural plant alkaloid berberine. J Clin Endocrinol Metab. 2008;93(7):2559-65. https://doi.org/10.1210/jc.2007-2404.

9. Zhu L, Zhang D, Zhu H, Zhu J, Weng S, Dong L, et al. Berberine treatment increases Akkermansia in the gut and improves high-fat diet-induced atherosclerosis in Apoe(-/-) mice. Atherosclerosis. 2018;268:117-26. https:// doi.org/10.1016/j.atherosclerosis.2017.11.023.

10. Habtemariam S. Berberine pharmacology and the gut microbiota: a hidden therapeutic link. Pharmacol Res. 2020;155:104722. https://doi.org/10.1016/j. phrs.2020.104722.

11. Zhang X, Zhao Y, Zhang M, Pang X, Xu J, Kang C, et al. Structural changes of gut microbiota during berberine-mediated prevention of obesity and insulin resistance in high-fat diet-fed rats. PLoS One. 2012;7(8):e42529. https://doi.org/10.1371/journal.pone.0042529. 
12. Yue SJ, Liu J, Wang AT, Meng XT, Yang ZR, Peng C, et al. Berberine alleviates insulin resistance by reducing peripheral branched-chain amino acids. Am J Physiol Endocrinol Metab. 2019;316(1):E73-85. https://doi.org/10.1152/a jpendo.00256.2018.

13. Sun R, Yang N, Kong B, Cao B, Feng D, Yu X, et al. Orally administered berberine modulates hepatic lipid metabolism by altering microbial bile acid metabolism and the intestinal FXR signaling pathway. Mol Pharmacol. 2017;91(2):110-22. https://doi.org/10.1124/mol.116.106617.

14. Liu D, Zhang Y, Liu Y, Hou L, Li S, Tian H, et al. Berberine modulates gut microbiota and reduces insulin resistance via the TLR4 signaling pathway. Exp Clin Endocrinol Diabetes. 2018;126(8):513-20. https://doi.org/10.1055/s0043-125066.

15. Cui HX, Hu YN, Li JW, Yuan K. Hypoglycemic mechanism of the berberine organic acid salt under the synergistic effect of intestinal flora and oxidative stress. Oxid Med Cell Longev. 2018;2018:8930374.

16. Wang Y, Shou JW, Li XY, Zhao ZX, Fu J, He CY, et al. Berberine-induced bioactive metabolites of the gut microbiota improve energy metabolism. Metabolism. 2017;70:72-84. https://doi.org/10.1016/j.metabol.2017.02.003.

17. Imenshahidi $\mathrm{M}$, Hosseinzadeh $\mathrm{H}$. Berberine and barberry (Berberis vulgaris): a clinical review. Phytother Res. 2019;33(3):504-23. https://doi.org/10.1002/ ptr.6252.

18. Chen Y-X, Gao Q-Y, Zou T-H, Wang B-M, Liu S-D, Sheng J-Q, et al. Berberine versus placebo for the prevention of recurrence of colorectal adenoma: a multicentre, double-blinded, randomised controlled study. The Lancet Gastroenterology \& Hepatology. 2020;5(3):267-75. https://doi.org/10.1016/ S2468-1253(19)30409-1.

19. Zhang LS, Zhang JH, Feng $R$, Jin XY, Yang FW, Ji ZC, et al. Efficacy and safety of berberine alone or combined with statins for the treatment of hyperlipidemia: a systematic review and meta-analysis of randomized controlled clinical trials. Am J Chin Med. 2019;47(4):751-67. https://doi.org/1 $0.1142 /$ S0192415X19500393.

20. Feng R, Shou JW, Zhao ZX, He CY, Ma C, Huang M, et al. Transforming berberine into its intestine-absorbable form by the gut microbiota. Sci Rep. 2015;5(1):12155. https://doi.org/10.1038/srep12155.

21. Ebrahimi ZS, Nasli-Esfahani E, Nadjarzade A, Mozaffari-Khosravi H. Effect of symbiotic supplementation on glycemic control, lipid profiles and microalbuminuria in patients with non-obese type 2 diabetes: a randomized, double-blind, clinical trial. J Diabetes Metab Disord. 2017;16(1): 23. https://doi.org/10.1186/s40200-017-0304-8.

22. Kijmanawat A, Panburana P, Reutrakul S, Tangshewinsirikul C. Effects of probiotic supplements on insulin resistance in gestational diabetes mellitus: a double-blind randomized controlled trial. J Diabetes Investig. 2019;10(1): 163-70. https://doi.org/10.1111/jdi.12863.

23. Gomes AC, de Sousa RG, Botelho PB, Gomes TL, Prada PO, Mota JF. The additional effects of a probiotic mix on abdominal adiposity and antioxidant status: a double-blind, randomized trial. Obesity (Silver Spring). 2017;25(1):30-8. https://doi.org/10.1002/oby.21671.

24. Kouchaki E, Tamtaji OR, Salami M, Bahmani F, Daneshvar Kakhaki R, Akbari E, et al. Clinical and metabolic response to probiotic supplementation in patients with multiple sclerosis: a randomized, double-blind, placebocontrolled trial. Clin Nutr. 2017;36(5):1245-9. https://doi.org/10.1016/j.clnu.2 016.08.015.

25. Famouri F, Shariat Z, Hashemipour M, Keikha M, Kelishadi R. Effects of probiotics on nonalcoholic fatty liver disease in obese children and adolescents. J Pediatr Gastroenterol Nutr. 2017;64(3):413-7. https://doi.org/1 $0.1097 / M P G .0000000000001422$.

26. Ming J, Xu S, Liu C, Liu X, Jia A, Ji Q. Effectiveness and safety of bifidobacteria and berberine in people with hyperglycemia: study protocol for a randomized controlled trial. Trials. 2018;19(1):72. https://doi.org/10.11 86/s13063-018-2438-5.

27. Truong DT, Franzosa EA, Tickle TL, Scholz M, Weingart G, Pasolli E, et al. MetaPhIAn2 for enhanced metagenomic taxonomic profiling. Nat Methods. 2015;12(10):902-3. https://doi.org/10.1038/nmeth.3589.

28. Li J, Jia H, Cai $X$, Zhong H, Feng Q, Sunagawa $S$, et al. An integrated catalog of reference genes in the human gut microbiome. Nat Biotechnol. 2014; 32(8):834-41. https://doi.org/10.1038/nbt.2942.

29. Patil KR, Nielsen J. Uncovering transcriptional regulation of metabolism by using metabolic network topology. Proc Natl Acad Sci U S A. 2005;102(8): 2685-9. https://doi.org/10.1073/pnas.0406811102.

30. Liang $Y, X u X$, Yin M, Zhang $Y$, Huang $L$, Chen $R$, et al. Effects of berberine on blood glucose in patients with type 2 diabetes mellitus: a systematic literature review and a meta-analysis. Endocr J. 2019;66(1):51-63. https://doi. org/10.1507/endocrj.EJ18-0109.

31. Zhang Y, Gu Y, Ren H, Wang S, Zhong H, Zhao X, et al. Gut microbiomerelated effects of berberine and probiotics on type 2 diabetes (the PREMOTE study). Nat Commun. 2020;11(1):5015. https://doi.org/10.1038/ s41467-020-18414-8.

32. Chen J, Wang R, Li XF, Wang RL. Bifidobacterium adolescentis supplementation ameliorates visceral fat accumulation and insulin sensitivity in an experimental model of the metabolic syndrome. Br J Nutr. 2012;107(10):1429-34. https://doi.org/10.1017/S0007114511 004491.

33. Qin J, Li Y, Cai Z, Li S, Zhu J, Zhang F, et al. A metagenome-wide association study of gut microbiota in type 2 diabetes. Nature. 2012; 490(7418):55-60. https://doi.org/10.1038/nature11450.

34. Zhang $X$, Zhao $Y, X u$ J, Xue Z, Zhang M, Pang $X$, et al. Modulation of gut microbiota by berberine and metformin during the treatment of high-fat diet-induced obesity in rats. Sci Rep. 2015;5(1):14405. https://doi.org/10.103 8/srep14405.

35. Tiderencel KA, Hutcheon DA, Ziegler J. Probiotics for the treatment of type 2 diabetes: a review of randomized controlled trials. Diabetes Metab Res Rev. 2020;36(1):e3213. https://doi.org/10.1002/dmrr.3213.

36. Kong WJ, Vernieri C, Foiani M, Jiang JD. Berberine in the treatment of metabolism-related chronic diseases: a drug cloud (dCloud) effect to target multifactorial disorders. Pharmacol Ther. 2020;209:107496. https://doi.org/1 0.1016/j.pharmthera.2020.107496.

37. Zmora N, Zilberman-Schapira G, Suez J, Mor U, Dori-Bachash M, Bashiardes $\mathrm{S}$, et al. Personalized gut mucosal colonization resistance to empiric probiotics is associated with unique host and microbiome features. Cell. 2018;174(6):1388-405 e21. https://doi.org/10.1016/j.cell.2 018.08.041.

38. Liu Y, Wang Y, Ni Y, Cheung CKY, Lam KSL, Wang Y, et al. Gut microbiome fermentation determines the efficacy of exercise for diabetes prevention. Cell Metab. 2020;31(1):77-91 e5. https://doi.org/10.1 016/j.cmet.2019.11.001.

39. Tanaka M, Sanefuji M, Morokuma S, Yoden M, Momoda R, Sonomoto K, et al. The association between gut microbiota development and maturation of intestinal bile acid metabolism in the first $3 \mathrm{y}$ of healthy Japanese infants. Gut Microbes. 2020;11(2):205-16. https://doi.org/10.1080/19490976.2019.1 650997.

40. Tanaka M, Sanefuji M, Morokuma S, Yoden M, Momoda R, Sonomoto K, et al. The association between gut microbiota development and maturation of intestinal bile acid metabolism in the first $3 \mathrm{y}$ of healthy Japanese infants. Gut Microbes. 2019:1-12.

41. Ethanic M, Stanimirov B, Pavlovic N, Golocorbin-Kon S, Al-Salami H, Stankov K, et al. Pharmacological applications of bile acids and their derivatives in the treatment of metabolic syndrome. Front Pharmacol. 2018;9:1382.

42. Hoffmann TW, Pham HP, Bridonneau C, Aubry C, Lamas B, MartinGallausiaux $C_{\text {, }}$ et al. Microorganisms linked to inflammatory bowel disease-associated dysbiosis differentially impact host physiology in gnotobiotic mice. ISME J. 2016;10(2):460-77. https://doi.org/10.1038/ ismej.2015.127.

43. Williams Brianna B, Van Benschoten AH, Cimermancic P, Donia Mohamed S, Zimmermann $M$, Taketani $M$, et al. Discovery and characterization of gut microbiota decarboxylases that can produce the neurotransmitter tryptamine. Cell Host Microbe. 2014;16(4):495-503. https://doi.org/10.1016/j. chom.2014.09.001.

44. Cervera-Tison M, Tailford LE, Fuell C, Bruel L, Sulzenbacher G, Henrissat B, et al. Functional analysis of family $\mathrm{GH} 36$ alpha-galactosidases from Ruminococcus gnavus E1: insights into the metabolism of a plant oligosaccharide by a human gut symbiont. Appl Environ Microbiol. 2012; 78(21):7720-32. https://doi.org/10.1128/AEM.01350-12.

45. Li CN, Wang X, Lei L, Liu MZ, Li RC, Sun SJ, et al. Berberine combined with stachyose induces better glycometabolism than berberine alone through modulating gut microbiota and fecal metabolomics in diabetic mice. Phytother Res. 2020;34(5):1166-74. https://doi.org/10.1002/ptr.6588.

46. Wu DN, Guan L, Jiang YX, Ma SH, Sun YN, Lei HT, et al. Microbiome and metabonomics study of quercetin for the treatment of atherosclerosis. Cardiovasc Diagn Ther. 2019;9(6):545-60. https://doi.org/10.21037/cdt.201 9.12.04. 
47. Zhang $W, X u J H, Y u T$, Chen QK. Effects of berberine and metformin on intestinal inflammation and gut microbiome composition in $\mathrm{db} / \mathrm{db}$ mice. Biomed Pharmacother. 2019;118:109131. https://doi.org/10.1016/j.biopha.201 9.109131.

48. Yu Y, Liu L, Wang X, Liu X, Liu X, Xie L, et al. Modulation of glucagonlike peptide-1 release by berberine: in vivo and in vitro studies. Biochem Pharmacol. 2010;79(7):1000-6. https://doi.org/10.1016/j.bcp.2 009.11.017.

49. Yu Y, Hao G, Zhang Q, Hua W, Wang M, Zhou W, et al. Berberine induces GLP-1 secretion through activation of bitter taste receptor pathways. Biochem Pharmacol. 2015;97(2):173-7. https://doi.org/10.1016/j.bcp.2015.07.012.

50. Holst JJ, Deacon CF. Glucagon-like peptide 1 and inhibitors of dipeptidyl peptidase IV in the treatment of type 2 diabetes mellitus. Curr Opin Pharmacol. 2004;4(6):589-96. https://doi.org/10.1016/j.coph.2 004.08.005.

51. Drucker DJ. The biology of incretin hormones. Cell Metab. 2006;3(3):153-65. https://doi.org/10.1016/j.cmet.2006.01.004

52. Houghton D, Stewart CJ, Day CP, Trenell M. Gut microbiota and lifestyle interventions in NAFLD. Int J Mol Sci. 2016;17(4):447. https://doi.org/10.3390/ ijms17040447.

53. Guevara-Cruz M, Flores-Lopez AG, Aguilar-Lopez M, Sanchez-Tapia M, Medina-Vera I, Diaz D, et al. Improvement of lipoprotein profile and metabolic endotoxemia by a lifestyle intervention that modifies the gut microbiota in subjects with metabolic syndrome. J Am Heart Assoc. 2019;8(17):e012401. https://doi.org/10.1161/JAHA.119.012401.

54. Fernandez-Murga ML, Olivares M, Sanz Y. Bifidobacterium pseudocatenulatum CECT 7765 reverses the adverse effects of diet-induced obesity through the gut-bone axis. Bone. 2020;141:115580. https://doi.org/1 0.1016/j.bone.2020.115580

55. Ming J, Yu X, Xu X, Wang L, Ding C, Wang Z, et al. Effectiveness and safety of Bifidobacterium and berberine in human hyperglycemia and their regulatory effect on the gut microbiota: a multi-center, double-blind, randomized, parallel-controlled study. Eur Nucleotide Arch. 2021; https:/ www.ebi.ac.uk/ena/browser/view/PRJEB44907.

\section{Publisher's Note}

Springer Nature remains neutral with regard to jurisdictional claims in published maps and institutional affiliations.

Ready to submit your research? Choose BMC and benefit from:

- fast, convenient online submission

- thorough peer review by experienced researchers in your field

- rapid publication on acceptance

- support for research data, including large and complex data types

- gold Open Access which fosters wider collaboration and increased citations

- maximum visibility for your research: over $100 \mathrm{M}$ website views per year

At $\mathrm{BMC}$, research is always in progress.

Learn more biomedcentral.com/submissions 